\title{
Resiliencia familiar: una revisión sobre artículos publicados en español
}

Family resilience: a review on articles in spanish

\author{
Héctor Bravo A. ${ }^{1}$ Judith López P. \\ Universidad Michoacana de San Nicolás de Hidalgo. Michoacán, México.
}

(RECIBIDO 30/07/2015, AcEPTADo 20/11/2015)

\begin{abstract}
RESUMEN
En este artículo se presenta una revisión temática sobre el estado del arte de los artículos de resiliencia familiar publicados en español. Partiendo de los factores de riesgo y protección se transita hacia una definición de resiliencia, así como al surgimiento del constructo. Así mismo, se describen los componentes del enfoque de resiliencia, tales como el modelo salutogénico, el ecológico y la psicología positiva. Se caracteriza a la resiliencia familiar con las aportaciones del enfoque sistémico y la teoría del estrés familiar; así como los principales procesos de resiliencia familiar. Se elaboran argumentos sobre la pertinencia del modelo para el abordaje de diversos fenómenos de actualidad. Se revisan las distintas publicaciones por tipos de estudio: investigaciones cualitativas, cuantitativas y artículos de revisión. Para finalizar, se elaboran conclusiones y recomendaciones emanadas de la revisión temática.
\end{abstract}

Palabras clave: factores de riesgo y protección, resiliencia, resiliencia familiar, procesos de resiliencia familiar.

\begin{abstract}
The current article presents a thematic review about the state of the art on family resilience articles published in spanish. Starting from the risk and protective factors, it moves toward a definition of resilience, as well as the emergence of the construct. Besides, there are described the components of the resilience approach, such as the salutogenic and ecological models and positive psychology. Family resilience is characterized from the systemic approach and the family stress theory, as well as the main family resilience processes. Is argued on the relevance of the model to approach to topical issues. Various publications are reviewed by types of study: cualitative and cuantitative researches, and review articles. Conclusions and recomendations emanating from the thematic review are presented to finish.
\end{abstract}

Keywords: risk and protective factors, resilience, family resilience, family resilience processes.

\footnotetext{
1 Maestro en Terapia Familiar. Estudiante del Doctorado Interinstitucional en Psicología sede Universidad Michoacana de San Nicolás de Hidalgo. hector.bravo@valles.udg.mx.

Agradecimiento al Consejo Nacional de Ciencia y Tecnología (Conacyt) por la beca otorgada para Programas Nacionales
} de Posgrados de Calidad (PNPC) para estudios de doctorado. 


\section{METODOLOGÍA}

El objetivo del presente es realizar una revisión temática sobre el estado del arte de la resiliencia familiar con base en artículos de divulgación científica publicados en español. Se realizó una búsqueda bibliográfica a partir de las bases de datos EBSCO, PROQUEST, SAGE, SPRINGER, WILEY, REDALYC, DIALNET. Los criterios de selección fueron: artículos publicados en español que integraran explícitamente el término resiliencia familiar en su título. Los términos de búsqueda fueron: resiliencia familiar, resiliencia y familia.

\section{INTRODUCCIÓN}

Los estudios en psicología han integrado modelos complejos en sus investigaciones actuales para una mejor comprensión e intervención en su campo de acción. Se ha transcurrido de concebir los fenómenos de manera aislada a considerar interrelaciones, dentro de las que destacan los factores de riesgo y protección.

Los factores de riesgo son definidos como "todas aquellas condiciones sociales, económicas o biológicas, conductas o ambientes que están asociados con o causan un incremento de la susceptibilidad para una enfermedad específica, una salud deficiente o lesiones" (Cardozo y Dubini, 2005, p. 47). Por su parte, los factores protectores se refieren a "influencias que modifican, mejoran o alteran la respuesta de una persona a algún peligro que predispone a un resultado no adaptativo" (Rutter, 1985, como se citó en Kotliarenco, 1997, p. 12). Ambos factores involucran interacción y pueden ser una cualidad de la persona o una característica del contexto o la familia (Cardozo y Dubini, 2005; Kotliarenco, 1997).

En este sentido, el concepto de resiliencia ha cobrado ímpetu como modelo de investigación desde factores protectores y recursos de las personas, familias y comunidades.

\section{Definición de resiliencia}

Se entiende por resiliencia a la "capacidad de una persona para recobrarse de la adversidad fortalecida y dueña de mayores recursos. Se trata de un proceso activo de resistencia, autocorrección y crecimiento como respuesta a las crisis y desafíos de la vida" (Walsh, 2004, p. 26). Por su parte, Rutter (1999) define operacionalmente la resiliencia mencionando que "ha habido resultados positivos para alguien a pesar de que la situación de su experiencia mostrara un mayor riesgo a desarrollar patología" (p. 119). Así también, Grotberg (2001) menciona que "la resiliencia es la capacidad humana para enfrentar, sobreponerse y ser fortalecido o transformado por experiencias de adversidad" (p. 20).

Tres elementos son comunes a las diversas definiciones de resiliencia: la noción de adversidad, como la muerte de un ser querido; la adaptación positiva ante tal adversidad; y el proceso que hace referencia a la interacción entre una multiplicidad 
de factores de riesgo y protección, tales como los familiares, afectivos, cognitivos, biológicos, socioeconómicos y culturales (Infante, 2006).

\section{Surgimiento del constructo}

El concepto resiliencia es relativamente nuevo en psicología, y ha cobrado interés en las últimas décadas (Rutter, 1999). Su aparición se remonta a las investigaciones pioneras de Werner (García-Vesga y Domínguez-De la Ossa, 2013), quien llevó a cabo en Hawai una investigación longitudinal dando seguimiento después de 30 años a 500 niños en condiciones de riesgo y precariedad, considerados con un pronóstico desfavorable. El principal hallazgo fue que, de manera contraria a lo propuesto por las teorías deterministas, no todas las personas que habían experimentado algún suceso traumático o condiciones adversas presentaban complicaciones, sino que una parte de ellas resolvían sus problemáticas de forma exitosa.

La interrogante subyacente al concepto de resiliencia es: ¿qué hace que ante ciertas adversidades algunas personas puedan superarlas y adaptarse, mientras que otras no logran hacerlo?

Los primeros estudios sobre resiliencia se caracterizan por tratar de identificar las características que distinguen a personas que superan adversidades de aquellas que no logran hacerlo, es decir, cualidades de los sujetos para enfrentar adversidades. De este modo, la resiliencia es entendida como conjunto de rasgos inherentes a la persona que le permite salir adelante ante el estrés psicosocial.

Desde la segunda generación de estudios sobre resiliencia, a mediados de los noventa, las investigaciones centran su atención en los procesos dinámicos entre las cuestiones individuales, familiares y comunitarias.

\section{Componentes}

El enfoque de resiliencia se enriquece de distintas aproximaciones:

El modelo salutogénico de Antonovsky (1996), orientado hacia el bienestar y posibilidades de personas y familias, centra el énfasis no en la enfermedad, sino en la salud y el bienestar, su origen, realce y mantenimiento; la forma en que la percepción de las experiencias de adversidad vividas tiene una influencia positiva en la salud (Taylor, 2004; Lindstrom y Eriksson, 2006; Hernán y Lineros, 2010).

El modelo ecológico supone que el desarrollo evolutivo de los seres humanos se genera en un sistema de ambientes anidados uno dentro de otro e interconectados entre sí, en el que todos influyen en distintos modos (Bronfenbrenner, 1987; Mc Guckin y Minton, 2014). Los sistemas o círculos de influencia son: a) microsistema, compuesto en primer lugar por los padres, maestros y pares; b) mesosistema, es el siguiente nivel, donde se encuentran el vecindario, trabajo, iglesia, etc.; c) exosistema, implica aquellos espacios en interrelación que tienen lugar fuera de la presencia directa del sujeto pero que ejercen una influencia en él, como el trabajo 
de los padres para un infante; d) macrosistema, referido a los grandes sistemas ideológicos que repercuten en la vida cotidiana, tales como cultura, economía y política; y e) cronosistema que se transita a lo largo del desarrollo en el tiempo (Brendtro, 2006).

La psicología positiva, por su parte, trata sobre el estudio de las experiencias subjetivas valoradas, como son el bienestar y la satisfacción referidas al pasado; esperanza y optimismo sobre el futuro, y el fluir y la felicidad en el presente. A nivel individual se refiere a los rasgos positivos como la capacidad de amar o las habilidades sociales; a nivel grupal, trata sobre las virtudes que mueven a los individuos hacia la ciudadanía, como la responsabilidad y la tolerancia (Seligman y Csikszentmihalyi, 2000).

\section{Resiliencia familiar}

El enfoque de resiliencia familiar se conforma de la confluencia entre las aportaciones de la resiliencia individual, enriquecida por la psicopatología del desarrollo, y el estudio de la familia, alimentado de la práctica clínica. Se trata de una orientación centrada en las fortalezas, que se aleja de los modelos basados en el déficit (Nichols, 2013).

La perspectiva sistémica concibe a la familia como un sistema en el que los elementos que la conforman, es decir, sus integrantes, se relacionan de tal manera que ejercen interacciones recíprocas, donde la conducta de un miembro influye y es influida por los demás elementos del sistema (Minuchin, 1986).

La unidad familiar, concebida como una cualidad sistémica compartida, permite pasar de lo individual a lo familiar, tal como mencionan Hewley y DeHaan:

Quizá la mayor contribución en la distinción conceptual, en la literatura sobre resiliencia familiar en ciernes, ha sido el desarrollo de la noción de ethos familiar (es decir, esquema, visión del mundo, sentido de coherencia), la cual intenta describir una serie de valores y actitudes compartidos, sostenida por la unidad familiar que sirve como eje de su resiliencia (1996, p. 63).

El enfoque de resiliencia familiar también se enriquece de la teoría del estrés familiar. El modelo de respuesta de ajuste y adaptación familiar implica el balance entre la interacción de demandas y capacidades familiares, mediatizado por los significados atribuidos. Cuando las demandas superan la capacidad de la familia, esta entra en un estado de desorganización llamado crisis, a partir del cual se ponen en marcha procesos emergentes que incrementan las capacidades o cambian los significados para adaptarse adecuadamente (Patterson, 2002).

El estrés y los retos se consideran como oportunidades para fomentar la curación y el crecimiento (Black y Lobo, 2008). Se transitó de concebir la familia solo como contexto de desarrollo de resiliencia individual a percibirla como una unidad de procesos resilientes en sí misma (Nichols, 2013). De este modo: 
La expresión 'resiliencia familiar' designa los procesos de superación y adaptación que tienen lugar en la familia como unidad funcional. La perspectiva sistémica permite comprender de qué manera los procesos familiares moderan el estrés y posibilitan a las familias afrontar penurias prolongadas y dejar atrás las situaciones de crisis (Walsh, 2004, p. 40).

Desde la teoría del estrés familiar, Hawley y DeHaan (1996) sostienen que:

La resiliencia familiar describe la trayectoria que una familia sigue, sobre cómo se adapta y prospera frente al estrés, tanto en el presente como a través del tiempo (...) dependiendo del contexto, nivel de desarrollo, combinación interactiva de factores de riesgo y protección, y el panorama familiar compartido (p. 65).

Tres procesos fundamentales dan soporte a la resiliencia familiar: sistema de creencias, procesos organizacionales y procesos de comunicación. Los principales factores de protección familiar que se destacan son: apego adecuado y apoyo, disciplina y monitorización, calidez y apoyo apropiados (Walsh, 2003, 2004). Para Patterson (2002), los procesos familiares de protección son: cohesión familiar, flexibilidad familiar, comunicación familiar y significados familiares. Black y Lobo (2008), por su parte, proponen como factores prominentes de resiliencia familiar: un panorama positivo; espiritualidad; acuerdos entre los miembros de la familia; flexibilidad; comunicación familiar; administración financiera; tiempo en familia; recreación compartida; rutinas y rituales; y redes de apoyo.

Algunos modelos centran la resiliencia familiar en los factores protectores, mientras que otros se concentran en los de recuperación. Los factores protectores facilitan el ajuste, mantienen la seguridad, el funcionamiento y completan las tareas de desarrollo; mientras que los factores de recuperación promueven la habilidad de adaptarse en situaciones de crisis (Black y Lobo, 2008).

Así pues, existe una perspectiva que defiende que para ser resiliente es necesario enfrentar riesgos significativos: a) continuos o crónicos, como la pobreza, b) exposición a un evento traumático o adversidad severa, tal como la guerra, o c) una combinación de ambos. La otra posición mantiene que todo aquel que funcione competentemente debe ser considerado resiliente puesto que la vida es suficientemente desafiante para crear exposición a los riesgos. La perspectiva del riesgo significativo surge de investigadores con poblaciones de riesgo, mientras que la perspectiva de la vida como riesgo, surge de profesionales interesados en las fortalezas de los individuos y las familias. Ambas perspectivas están relacionadas (Patterson, 2002).

Sumariamente, la pertinencia del modelo de resiliencia familiar estriba en tres cuestiones fundamentales.

Primero, a partir de sus componentes engloba en un solo constructo toda una variedad de cuestiones familiares, tales como cohesión y adaptabilidad, sistemas de creencias, formas de organización, comunicación y resolución de problemas.

En segundo término, desde esta perspectiva se consideran tanto los contextos o sistemas más amplios, como la evolución de las personas y familias. De esta 
forma, el modelo es pertinente para el estudio de distintos fenómenos, al tratarse de un modelo abarcativo.

Por último, "en lugar de factores de riesgo y resultados problemáticos, el trabajo con resiliencia se preocupa por las fortalezas familiares, la adaptación positiva y lo que lleva a mejores resultados para la familia en su conjunto" (Fitzgerald, 2010, p. 7). Lo cual permite abonar en acciones que contribuyan al fortalecimiento de las familias.

\section{REVISIÓN}

Existe un interés creciente en los países de habla hispana por el modelo de resiliencia familiar, aun en desarrollo. En la literatura disponible en español se encontraron 13 publicaciones realizadas del 2003 al 2013 que consideran explícitamente en su título el término resiliencia familiar y uno sobre pautas parentales resilientes. Seis son de tipo cualitativo, dos cuantitativas y cinco de revisión. A continuación se presentan los diversos estudios por tipo de publicación y orden cronológico, destacando los procesos de resiliencia familiar encontrados en cada artículo.

Tabla 1. Tipo de publicación sobre resiliencia familiar.

\begin{tabular}{lll}
\hline \multicolumn{1}{c}{ Cualitativos } & \multicolumn{1}{c}{ Cuantitativos } & \multicolumn{1}{c}{ Revisión } \\
\hline González (2004) & Kotliarenco, Muñoz y Muzzio (2012) & Villalba (2003) \\
Domínguez y Godín (2007) & $\begin{array}{l}\text { Ramírez-Osorio y Hernández- } \\
\text { Mendoza (2012) }\end{array}$ & $\begin{array}{l}\text { (2010) } \\
\text { De Andradiarenco y Da Cruz } \\
\text { Castiblanco y Lora (2008) }\end{array}$ \\
Borges y Silva (2010) & & Herscovici (2011) \\
Pereira (2010) & & Grau (2013) \\
Fernández, Parra y & & \\
Torralba (2012) & & \\
\hline
\end{tabular}

\section{ESTUDIOS CUALITATIVOS}

González (2004) llevó a cabo en Colombia una investigación socio-construccionista cuyo objetivo fue comprender las transformaciones por las que atraviesan las familias desplazadas hacia Bogotá por la violencia. Las técnicas utilizadas fueron las entrevistas semiestructuradas y diarios de campo.

Se define resiliencia como "el proceso en el cual los individuos y los grupos sociales, dadas ciertas condiciones favorables en su contexto, desarrollan progresivamente habilidades o recursos que les permiten afrontar positivamente situaciones adversas" (González, 2004, p. 124).

Las principales fortalezas que desarrollan las familias desplazadas son: reflexiones sobre el valor de la vida y sentido de la experiencia; reconstrucción de proyectos 
de vida a partir del nuevo sentido atribuido; constancia en el logro de metas, sobre todo en mujeres cabeza de familia; actitud empática en el reconocimiento de emociones y sentimientos de otros; unión familiar a pesar de la distancia; solución conjunta de problemas cotidianos; buen humor como forma de comunicación; generosidad hacia otros desplazados; construcción de nuevas redes de apoyo entre parientes y vecinos (González, 2004).

Domínguez y Godín (2007) investigan los desplazamientos en Colombia como problemática social producto de la violencia. La aproximación se realiza desde la teoría de resiliencia de Cyrulnik (1999, como se citó en Domíguez y Godín, 2007).

El método utilizado fue estudio de casos, a partir de entrevistas semiestructuradas, relatos de vida y observación participante; así también se aplicó la Escala de Resiliencia en Niños (ESRENI) y el esquema de la "casita" de Vanistendael (2002, como se citó en Domínguez y Godín, 2007).

Se definen resiliencia como las "potencialidades y recursos de los seres humanos para afrontar y sobrepasar una situación extrema" (Domínguez y Godín, 2007, p. 156). Como características resilientes en las familias desplazadas destacan dos cuestiones. Primero, "la habilidad para planear metas y expectativas por un futuro mejor, solicitar y dar ayuda y la fe en un ser superior" (Domínguez y Godín, 2007, p. 176). En segundo lugar, que el rol de la mujer moviliza la resiliencia de la familia ante el golpe del desplazamiento.

Castiblanco y Lora (2008) realizaron en Colombia, una investigación-intervención cuyo objetivo fue generar contextos que permitan identificar, comprender y potenciar pautas de relación y estrategias parentales resilientes en un grupo de familias con hijos adolescentes. Fue un diseño cualitativo con el uso de guiones para entrevistas a familias con hijos adolescentes en la Fundación de la Universidad de la Familia, de corte religioso en Bogotá. Dentro de la fundación se trabaja con padres a manera de grupos de autoayuda (Castiblanco y Lora, 2008).

La resiliencia familiar es entendida como un ambiente "clave para que los individuos desarrollen la capacidad de sobreponerse a la adversidad, se adapten a las presiones y problemas que enfrentan y desarrollen las competencias -social, académica y vocacional- necesarias para salir adelante en la vida" (Castiblanco y Lora, 2008, p. 41). Como principales pautas resilientes resultaron la corresponsabilidad, demostración de apoyo emocional, pautas de generación y reconocimiento de confianza, pautas de comunicación asertiva, flexibilidad e innovación y pautas de creatividad. Los autores concluyen sobre la importancia de involucrar a la familia como parte del trabajo de intervención (Castiblanco y Lora, 2008).

Desde la enfermería en Portugal, Borges y Silva (2010) realizaron una investigación sobre el cuidado de pacientes con enfermedades crónicas, en relación a la esperanza y la resiliencia familiar. Desde una aproximación cualitativa utilizan como método las prácticas apreciativas, un proceso activo en el que todos los participantes contribuyen, retomando sus potencialidades frente a la adversidad. 
Las autoras retoman la definición de resiliencia familiar, elaborada por Walsh (2005), como un "proceso de enfrentamiento y adaptación de la familia como una unidad funcional" (como se citó en Borges y Silva, 2010, p. 253); haciendo énfasis en el desarrollo de competencias familiares.

Las principales prácticas que impulsan la transformación en las familias son la "recreación de historias de esperanza, superación y recuperación de la adversidad, como factor protector y promotor de esperanza" (Borges y Silva, 2010, p. 256).

Pereira (2010), médico psiquiatra, desde el contexto español, elabora un análisis sobre los factores de resiliencia familiar a partir de un estudio de caso de una joven cuya madre se suicidó cuando ella tenía tres años. Siguiendo a Walsh (2004), define la resiliencia familiar como "la capacidad de una familia para recuperarse de circunstancias adversas y salir de ellas fortalecida y con mayores recursos para afrontar otras dificultades de la vida" (como se citó en Pereira, 2010, p. 18).

Como principales factores protectores de resiliencia familiar el autor distingue: la integración, la comunicación y la adaptación que pueda presentar la familia ante los acontecimientos estresantes, así como la significación del hecho traumático en el núcleo familiar como mecanismo para dotar de sentido la adversidad y de este modo superarla o sucumbir ante ella.

En un artículo español, Fernández, Parra y Torralba (2012) destacan como principales puntos de encuentro entre el trabajo social con familias y el modelo de resiliencia familiar: el modelo bio-psico-social; la visión hacia las fortalezas y capacidades de las familias para hacer frentele a las adversidades; y la importancia de la estructuración de una red de apoyo. Conceptualizan la resiliencia familiar como "la capacidad de auto reparación de la propia familia" (Fernández, et al., 2012 , p. 96), a partir de los procesos que se movilizan por los desafíos, así como el momento evolutivo que enfrenta la familia.

Presentan resultados de una investigación-acción cooperativa cuyo objetivo fue "generar conocimiento sobre la capacidad de resiliencia de las familias de cogida extensa para mejorar el proceso de adaptación de niños acogidos en la familia" (Fernández, et al., 2012, p. 98). Participaron 62 familias, realizando 64 entrevistas antes y después del programa, así también se realizaron 8 grupos de discusión.

Los principales hallazgos en relación a factores de protección fueron "la iniciativa para satisfacer las necesidades, la actitud, la conciencia de problema y la motivación para el cambio de las familias vulnerables en cualquier proceso de trabajo de mejora de las competencias parentales" (Fernández, et al., 2012, p. 99). Los grupos de discusión permitieron el intercambio de experiencias, reconocimiento y fortalecimiento recíproco de las familias, aumentando la cohesión y comprensión de los problemas (Fernández, et al., 2012). 
Tabla 2. Estudios cualitativos sobre resiliencia familiar.

\begin{tabular}{|c|c|c|}
\hline Autor(es) & Contexto & Procesos resilientes \\
\hline González (2004) & $\begin{array}{l}\text { Familias } \\
\text { desplazadas }\end{array}$ & $\begin{array}{l}\text { Sentido y proyectos de vida, logro de metas, actitud } \\
\text { empática, unión familiar, solución conjunta de problemas, } \\
\text { buen humor, generosidad y redes de apoyo. }\end{array}$ \\
\hline $\begin{array}{l}\text { Domínguez y } \\
\text { Godín (2007) }\end{array}$ & $\begin{array}{l}\text { Familias } \\
\text { desplazadas }\end{array}$ & $\begin{array}{l}\text { Habilidad para planear metas, expectativas en un futuro } \\
\text { mejor, solicitar y dar ayuda, fe en un ser superior y rol de } \\
\text { mujer moviliza resiliencia de la familia, }\end{array}$ \\
\hline $\begin{array}{l}\text { Castiblanco y } \\
\text { Lora (2008) }\end{array}$ & $\begin{array}{l}\text { Padres } \\
\text { con hijos } \\
\text { adolescentes }\end{array}$ & $\begin{array}{l}\text { Corresponsabilidad en demostración de apoyo emocional, } \\
\text { pautas de generación y reconocimiento de confianza, pautas } \\
\text { de comunicación asertiva, flexibilidad e innovación y pautas } \\
\text { de creatividad. }\end{array}$ \\
\hline $\begin{array}{l}\text { Borges y Silva } \\
(2010)\end{array}$ & $\begin{array}{l}\text { Padres con } \\
\text { un hijo con } \\
\text { enfermedad } \\
\text { crónica }\end{array}$ & $\begin{array}{l}\text { Recreación de historias de esperanza, superación y } \\
\text { recuperación de la adversidad, como factor protector y } \\
\text { promotor de esperanza. }\end{array}$ \\
\hline Pereira (2010) & $\begin{array}{l}\text { Psicoterapia } \\
\text { (suicidio de la } \\
\text { madre) }\end{array}$ & $\begin{array}{l}\text { Integración, comunicación, adaptación y significación de la } \\
\text { familia ante acontecimientos estresantes. }\end{array}$ \\
\hline $\begin{array}{l}\text { Fernández, Parra } \\
\text { y Torralba (2012) }\end{array}$ & $\begin{array}{l}\text { Familias de } \\
\text { acogida de } \\
\text { niños }\end{array}$ & $\begin{array}{l}\text { Iniciativa en satisfacer necesidades, actitud, conciencia } \\
\text { de problema, motivación para el cambio, mejora de } \\
\text { competencias parentales. }\end{array}$ \\
\hline
\end{tabular}

\section{ESTUDIOS CUANTITATIVOS}

Kotliarenco, Muñoz y Gómez (2012) realizaron en Chile un estudio comparativo de diseño transversal, descriptivo y correlacional, no experimental, cuyo objetivo fue estudiar la presencia o ausencia de resiliencia relacional en familias con hijos de entre 0 y 3 años de edad, sometidas a adversidad social y económica, específicamente aquellas identificadas como multiproblemáticas.

Los autores retoman la definición de resiliencia familiar de Gómez y Kotliarenco (2010), entendida como "conjunto de procesos de reorganización de significados y comportamientos que activa una familia sometida a estrés, para recuperar y mantener niveles óptimos de funcionamiento y bienestar, equilibrar recursos y necesidades familiares, y aprovechar las oportunidades de su entorno" (p. 124).

Definiendo operacionalmente resiliencia familiar a partir del funcionamiento adecuado/fortalecido arrojado por el ítem "interacción familiar" de la Escala de Evaluación Familiar Integral de Carolina del Norte (NCFAS-G), los autores destacan como hallazgo principal que:

Las familias con niños en infancia temprana que destinan tiempo a la recreación y el ocio, a jugar en familia, presentan 20 veces más probabilidades de desarrollar resiliencia familiar relacional bajo condiciones de adversidad social y económica. Jugar en familia es la llave que despertó la resiliencia en estas familias" (Kotliarenco, Muñoz y Gómez, 2012, p. 20). 
Otras variables asociadas de forma significativa son: la dinámica familiar saludable, el apego, la comunicación con la figura paterna, la relación cooperativa con el cuidador, el buen trato en pareja, las rutinas y rituales familiares. Como coralario a estas variables se destaca que "relación y organización podrían proponerse entonces como los pilares constitutivos de la resiliencia familiar" (Kotliarenco, Muñoz y Gómez, 2012, p. 21).

Ramírez-Osorio y Hernández-Mendoza (2012) realizaron una investigación para determinar la correlación entre resiliencia familiar, depresión y ansiedad en adolescentes de nivel socioeconómico bajo en Chimalhuacán, estado de México; para lo cual se realizó un estudio transversal con una muestra no probabilística de 100 adolescentes de entre 13 y 16 años, de tres escuelas secundarias.

Los autores definen la resiliencia familiar como "la adaptación inmediata y mediata de todos sus integrantes, así como en la supervivencia y bienestar de la unidad familiar" (Ramírez-Osorio y Hernández-Mendoza, 2012, p. 64).

De forma operacional la resiliencia familiar se definió con el Inventario de Detección de Recursos Familiares (IDERFAM) que consta de tres factores: patrones de organización, sistema de creencias, y comunicación familiar, distribuidos en 28 reactivos evaluados a través de una escala tipo Likert; posee un alpha de Cronbach de 0.947. También se utilizaron como instrumentos, un cuestionario de datos generales, el Inventario de Depresión de Beck (BDI) y, el Inventario de Ansiedad de Hamilton. El análisis de datos se realizó a partir del programa SPSS.

Los resultados principales arrojados fueron que existe una correlación significativa negativa (0.01) tanto entre resiliencia y depresión, como entre resiliencia y ansiedad, es decir, a mayor grado de resiliencia, menor grado de depresión y ansiedad. Los recursos familiares fungen como factores de protección ante la ansiedad y la depresión. La situación socioeconómica desfavorable es un factor de riesgo, más no determinante para la presencia de ansiedad y depresión.

Tabla 3. Estudios cuantitativos sobre resiliencia familiar.

\begin{tabular}{lll}
\hline \multicolumn{1}{c}{ Autor(es) } & \multicolumn{1}{c}{ Contexto } & \multicolumn{1}{c}{ Procesos resilientes } \\
\hline $\begin{array}{l}\text { Kotliarenco, Muñoz y } \\
\text { Muzzio (2012) }\end{array}$ & $\begin{array}{l}\text { Familias en } \\
\text { adversidad } \\
\text { socioeconómica con } \\
\text { hijos de entre 0 a } 3 \\
\text { años }\end{array}$ & $\begin{array}{l}\text { La recreación y el juego en familia es un } \\
\text { proceso proximal para el desarrollo de } \\
\text { resiliencia familiar en familias con niños de } \\
\text { entre 0 y tres años. }\end{array}$ \\
$\begin{array}{l}\text { Ramírez-Osorio y } \\
\text { Hernández-Mendoza } \\
(2012)\end{array}$ & $\begin{array}{l}\text { Depresión y ansiedad } \\
\text { en adolescentes }\end{array}$ & $\begin{array}{l}\text { A mayor grado de resiliencia, menor grado de } \\
\text { depresión y ansiedad. Los recursos familiares } \\
\text { fungen como factores de protección ante la } \\
\text { ansiedad y la depresión. }\end{array}$ \\
\hline
\end{tabular}




\section{Artículos de revisión}

La investigadora española Villalba (2003) retoma tres etapas en las investigaciones sobre resiliencia propuestas por Richardson (como se citó en Villalba, 2003) que van de conceptualizar la resiliencia como cualidades de la persona, pasando por entenderla como un proceso de influencias entre entorno e individuo, a suponer una energía o resiliencia en los seres humanos que proviene de fuentes ecológicas.

La resiliencia familiar se define como procesos que permiten a la familia como unidad hacer frente a las crisis dentro del contexto en que se desenvuelven desde una visión evolutiva a lo largo del ciclo vital. Los principales factores que promueven la resiliencia familiar son: cohesión y estabilidad; fuentes de apoyo y límites claros; género masculino; relación con pares; y autoeficacia (Villalba, 2003).

Se mencionan distintos enfoques en resiliencia familiar: el sistémico de Dallos (1996 como se citó en Villalba, 2003), que asigna más importancia a los procesos que a la forma de la familia. El enfoque multidimensional de Falicov (1998, como se citó en Villalba, 2003) que destaca la importancia del contexto, la persona y cuestiones socioeconómicas y culturales en la configuración de la familia y la resolución de conflictos. La teoría del estrés de Patterson (2002 como se citó en Villalba, 2003) destaca la subjetividad familiar ante las situaciones estresantes y el papel de las situaciones de protección en la resiliencia familiar.

Goméz y Kotliarenco (2010) elaboran una revisión temática desde Chile a través de varias bases de datos con conceptos como: resiliencia familiar, estrés familiar, factores de riesgo, factores protectores, adaptación familiar, salud familiar y funcionamiento familiar, en español e inglés.

Proponen una definición propia de resiliencia familiar como "el conjunto de procesos de reorganización de significados y comportamientos que activa una familia sometida a estrés, para recuperar y mantener niveles óptimos de funcionamiento y bienestar, equilibrar recursos y necesidades familiares, y aprovechar las oportunidades de su entorno" (Gómez y Kotliarenco, 2010, p. 124).

Destacan procesos de resiliencia transversales encontrados en los resultados de las investigaciones analizadas, distinguiendo los tipos de estudios según su metodología.

En tres investigaciones cualitativas sobre adversidad psicosocial y crianza positiva, desplazamiento familiar por violencia armada y enfermedad de cáncer de un hijo los procesos compartidos fueron: "la perseverancia en objetivos y actividades que beneficiaron la unidad familiar; el soporte o apoyo mutuo mediante una actitud empática con el otro; y la búsqueda activa de nuevas redes de apoyo" (Gómez y Kotliarenco, 2010, p. 117).

En estudios cuantitativos sobre resiliencia familiar se mencionan cinco investigaciones sobre: familias en riesgo psicosocial, familias con una hospitalización psiquiátrica, familias con escasos ingresos económicos, familias a lo largo del 
ciclo vital, y familias con un hijo autista. Los procesos de resiliencia familiar transversales que comparten los resultados de dichas investigaciones son: "generar espacios y procesos de comunicación en la familia; resolver conjuntamente los problemas; fortalecer la cohesión familiar y apoyo mutuo; enriquecer la calidad de la interacción padre-hijo; practicar y desarrollar las competencias parentales; y acceder a fuentes de apoyo social" (Gómez y Kotliarenco, 2010, p. 118).

Se destacan las aplicaciones del modelo en los campos de la terapia familiar, programas de intervención clínica, intervención psicosocial con familias multiproblemáticas, terapia ocupacional y salud mental, salud familiar y manejo de catástrofes naturales, terrorismo o crisis económicas. Por otra parte, ponen de manifiesto varios programas públicos sustentados en el modelo de resiliencia familiar implementados en Chile que han mostrado resultados favorables en cuanto a la promoción del desarrollo infantil temprano, prevención del maltrato infantil y atención en centros de salud familiar (Gómez y Kotliarenco, 2010).

En una revisión de estudios sobre familias brasileñas en condiciones de pobreza, De Andrade y Da Cruz (2011), reportan varias investigaciones:

En un estudio de caso en una familia en situación de riesgo, se verificó en la pareja el uso de estrategias de enfrentamiento basadas en un sistema de creencia en común a partir de la valoración de las relaciones interpersonales (Yunes y Szymanski, 2006, como se citó en De Andrade y Da Cruz, 2011).

En familias monoparentales se constató el apoyo de otros familiares, la transformación del funcionamiento familiar, creencias y valores en torno al estudio y al trabajo (Yunes et al., 2004, como se citó en De Andrade y Da Cruz, 2011).

Las autoras enfatizan que en Brasil existen investigaciones basadas en la propuesta ecológica de Bronfenbrenner. En consonancia con dicha propuesta Cecconello y Koller (2003, como se citó en De Andrade y Da Cruz, 2011), realizaron una investigación durante cuatro años con familias en riesgo, analizando los factores de riesgo y protección tanto a nivel intra como extrafamiliar. Se destacan como factores de protección las características personales de los miembros, la cohesión social y el apoyo conyugal y social.

A partir del modelo bioecológico del desarrollo humano, De Antoni (2005 como se citó en De Andrade y Da Cruz, 2011), dio seguimiento a 20 familias de nivel socioeconómico bajo con abuso intrafamiliar, el equipo participó en los contextos del hogar, hospital, colegio y una organización no gubernamental. Los factores de protección identificados fueron: características de los miembros de la familia, como talentos, la valoración del estudio y religiosidad, y otros factores que no fueron suficientes para evitar la violencia dada la falta de soporte social, programas de apoyo y políticas públicas.

Los principales procesos de resiliencia en las familias brasileñas que se destacan a partir de las investigaciones son: "vínculo, cohesión y creencias familiares como fuentes de apoyo ante la crisis" (De Andrade y Da Cruz, 2011, p. 52). 
Herscovici (2011), autora argentina, presenta un artículo de revisión temática en el que analiza el desarrollo de la resiliencia familiar a través de distintas etapas de investigación.

Las primeras investigaciones se centran en los factores de riesgo, a partir del cuestionamiento sobre por qué algunas personas ante situaciones desfavorables salen adelante mientras que otras en similares circunstancias no logran hacerlo. En esta etapa se atribuye la resiliencia a características de la personalidad.

Aun cuando la familia es percibida como fuente de riesgo se destacan como características de familias resilientes: el optimismo, fortalezas ante desafíos, clima afectuoso con límites claros, expresión de interés y amor, participación activa de los integrantes, capacidad autocrítica, escucha y aprendizaje, confianza, actividades conjuntas, resolución conjunta de problemas (Herscovici, 2011).

En una siguiente etapa se consideran los factores de riesgo y protección en relación a cuestiones individuales, familiares y socioculturales, "un inter-juego entre natura y nurtura" (Herscovici, 2011, p. 106). Diversos estudios intentaban determinar características de la familia que neutralizan la vulnerabilidad y el estrés. Lograron identificar como elementos básicos de la resiliencia familiar: "cohesión, flexibilidad, estabilidad, organización, buena comunicación entre los miembros de la familia, la reafirmación de un sistema de creencias comunes y buena capacidad de resolución de problemas" (Herscovici, 2011, p. 107).

Posteriormente las investigaciones se centraron en los factores de protección, específicamente en los procesos que estos ponen en marcha. Retomando las aportaciones del enfoque sistémico, ecológico y evolutivo se centraron en los procesos interactivos que definen la resiliencia familiar como la "habilidad para enfrentar y sobreponerse a los desafíos vitales disruptivos" (Herscovici, 2011, p. 108). Los procesos clave de resiliencia son: el sistema de creencias que permite dar coherencia a la adversidad a través de optimismo y esperanza; los procesos de organización a partir de la flexibilidad y la estabilidad y, por último, la habilidad de comunicación y solución de problemas (Walsh, 1998, como se citó en Herscovici, 2011).

En un artículo publicado en España, Grau (2013) describe los distintos enfoques sobre resiliencia familiar, enfatizando el contexto de familias con enfermedades crónicas pediátricas. Retomando las aportaciones de Walsh (1996 como se citó en Grau, 2013) define la resiliencia familiar como "los procesos interactivos que favorecen al individuo y a la familia en el transcurso del tiempo" (p. 96); la resolución de problemas se genera a partir de la cohesión, la comunicación y el sistema de creencias comunes.

El primer enfoque descrito es el de respuesta familiar de ajuste y adaptación, este sugiere que ante el estrés familiar hay pocos cambios en las formas de funcionamiento, recursos y estrategias de afrontamiento; al no ser suficientes los ajustes para superar el estrés, la familia entra en crisis, estado de desorganización que desemboca en la necesidad de cambios, por lo que se elabora una adaptación, en la 
que se cambian patrones de funcionamiento y se generan nuevos recursos de apoyo, estrategias de afrontamiento y una modificación de creencias, metas y valores.

La familia enfrenta situaciones estresantes en torno a la enfermedad: la naturaleza misma de la enfermedad, las reacciones del hijo, la familia, el entorno social y el sistema de salud. Ante tales fuentes de estrés se dispone de recursos como: las capacidades y actitudes del niño enfermo, características de la familia, comunidad y sistema comunitario. La autora recupera las aportaciones de McCubbin y de Patterson (2002; 2002 como se citó en Grau, 2013) sobre los factores de resiliencia familiar ante enfermedades crónicas, destacando "la rápida movilización y reorganización de la familia; el apoyo del equipo de cuidados sanitarios, de la familia extensa y de la comunidad; y los cambios de valores de la familia" (p. 199).

El siguiente enfoque descrito por Grau es el de resiliencia y procesos clave elaborado por Walsh (2002; 2003; Rolland y Walsh, 2006 como se citó en Grau, 2013); en el que se proponen tres procesos clave: el sistema de creencias, conformado por dotar de un sentido a la adversidad, la adopción de una perspectiva positiva y el desarrollo de valores de trascendencia y espiritualidad; los patrones organizativos por su parte consisten en la flexibilidad, la conectividad y los recursos sociales y económicos; y por último el proceso de comunicación y resolución de problemas que consta de la comunicación con claridad, expresión emocional abierta y la resolución de problemas de forma colaborativa.

Posteriormente se expone el enfoque del sistema familiar y enfermedad de Rolland (1994 como se citó en Grau, 2013), en el que se contemplan tres dimensiones: características de la enfermedad, considerando sí es aguda o gradual, progresiva o constante, recaídas, grado de incapacidad y amenaza a la existencia; fases de la enfermedad en las que se encuentran la crisis inicial, la crónica y la terminal; interacción entre el ciclo vital individual y familiar.

Como cuarto enfoque se presenta el ecológico y de desarrollo a partir de los planteamientos de Falicov, McGoldrick y Masten (1995, 2007; 2005; 2009 como se citó en Grau, 2013), donde la resiliencia familiar "depende de la pertenencia simultánea y su participación en múltiples contextos” (p. 202). En lo referente a la cuestión ecológica se consideran las relaciones con los diferentes contextos e instituciones, la cultura y el ciclo vital de la familia, considerando la singularidad de cada una de ellas. Por otro lado, el desarrollo se ve influido por el ciclo vital tanto familiar como de sus miembros, incluyendo la historia multigeneracional como elementos a considerar en cualquier intervención con enfermedades crónicas pediátricas.

Grau (2013) plantea algunos principios de intervención derivados de los enfoques sobre resiliencia familiar en relación a enfermedades crónico pediátricas: saber si la familia se encuentra en fase de ajuste o adaptación, conocer los factores protectores, claridad en los objetivos de intervención, contextualizar los procesos a partir de su evaluación, conocer la incidencia de la enfermedad y la manera de enfrentamiento de la familia, así como las relaciones con las instituciones sanitarias. De manera general los servicios centrados en la familia fortalecen sus 
estructuras, fomentan sus capacidades, movilizan recursos y apoyos y generan responsabilidad; desde una integración multidisciplinar entre médicos, psicólogos, maestros y trabajadores sociales.

\section{DISCUSIÓN Y CONCLUSIONES}

A partir de la revisión temática de artículos sobre resiliencia familiar publicados en español, podemos concluir algunas cuestiones cuantitativas, tales como:

El país con más publicaciones sobre resiliencia familiar es España (30.8\%), con un total de cuatro artículos de los trece encontrados (ver Tabla 4).

Tabla 4. Publicaciones por país.

\begin{tabular}{ccc}
\hline País & Cantidad & \% \\
\hline España & 4 & 30.8 \\
Colombia & 3 & 23.1 \\
Chile & 2 & 15.4 \\
Argentina & 1 & 7.7 \\
Brasil & 1 & 7.7 \\
México & 1 & 7.7 \\
Portugal & 1 & 7.7 \\
\hline
\end{tabular}

Entre el 2003 y el 2013 se publicaron 13 artículos sobre resiliencia familiar, siendo el 2010 y el 2012 los años con más publicaciones, con tres artículos por cada año, lo cual marca un incremento en las publicaciones totales en esta década, aun cuando no se sostiene el incremento (ver Tabla 5).

Tabla 5. Publicaciones por año.

\begin{tabular}{ccc}
\hline Año & Cantidad & $\%$ \\
\hline 2003 & 1 & 7.7 \\
2004 & 1 & 7.7 \\
2005 & 0 & 0 \\
2006 & 0 & 0 \\
2007 & 1 & 7.7 \\
2008 & 1 & 7.7 \\
2009 & 0 & 0 \\
2010 & 3 & 23.1 \\
2011 & 2 & 15.4 \\
2012 & 3 & 23.1 \\
2013 & 1 & 7.7 \\
\hline
\end{tabular}


En relación al tipo de artículo publicado se encontraron seis de tipo cualitativo, lo cual corresponde al $46.2 \%$, las investigaciones cuantitativas solamente representaron el $15.4 \%$ con dos publicaciones, mientras que los artículos de revisión constituyeron el $38.5 \%$ al contar con cinco artículos (ver Tabla 1).

A continuación se presentan algunas consideraciones tanto teóricas como metodológicas que vale la pena revisar, en aras de fortalecer el modelo de resiliencia familiar:

Es preciso aclarar que gran parte de los estudios referidos en los artículos revisados no suponen de manera explícita investigaciones conducentes a la resiliencia familiar de forma directa, sino a teorías complementarias como son las dirigidas a riesgo psicosocial, afrontamiento del estrés y desarrollo en el ciclo vital, de las cuales resultan procesos de resiliencia familiar. Las fronteras entre los estudios de resiliencia familiar propiamente y aquellas de las teorías complementarias es tan tenue que se pueden derivar enriquecedoras conclusiones sobre procesos familiares resilientes de dichas investigaciones.

Como una de las principales críticas se puede aducir que al parecer en algunas investigaciones se habla más de competencia que de resiliencia. Las competencias hacen alusión a cierta constancia subyacente y suponen respuestas homogéneas por parte de los individuos, lo cual no está supuesto en la resiliencia (Rutter, 2012). Por otra parte, no se define de manera clara que se entiende por competencias, al parecer se identifican con los recursos de la familia.

Uno de los puntos de encuentro principales en las distintas investigaciones se refiere al hacer a la familia partícipe de las reflexiones y decisiones sobre sus propias problemáticas. Así como el énfasis en el modelo ecológico de Bronfenbrenner (1987).

Existe una serie de consideraciones hacia las investigaciones sobre resiliencia familiar que no han sido resueltas, no solo en las publicaciones en español, sino a nivel general. Si bien la mayoría de los estudios encontrados en la presente revisión se circunscriben dentro de la metodología cualitativa, tal como es la tendencia actual de la investigación en resiliencia familiar; es necesario afinar algunos puntos que se describen a continuación.

Una de las principales cuestiones metodológicas se refiere a la operacionalización, y aunado a esto, específicamente a si se trata de una variable categórica (es o no es resiliente) o continua (varía en grados). Hawley y DeHaan (1996) proponen que sí se entiende como variable un elemento que posee dimensiones cambiantes e identificables y dado que la resiliencia familiar es considerada más como una trayectoria que la familia manifiesta a través de una variedad de puntos de estrés dentro del ciclo de vida, debe ser considerada como un constructo no variable.

Por su parte Card y Barnett (2015) plantean como solución al problema de si la resiliencia familiar es una cantidad (es más o menos resiliente) o si son tipos de familias, proponen que desde la visión de categoría se pueden hacer subgrupos a través de métodos empíricos de análisis (posición intermedia). Asimismo, pugnan 
por expectativas teóricas centradas en la persona, más que en la variable (Card y Barnett, 2015).

Uno de los principales problemas metodológicos que resultan en el estudio de la resiliencia familiar es la extrapolación de lo individual a lo familiar o viceversa. Por un lado existe un tipo de aproximación a la resiliencia familiar que reside en considerar las características de la familia, en cuanto a su estructura, funcionamiento y pautas relacionales, para después extrapolarlas a los individuos. Por otra parte, se encuentran intentos empíricos de aplicar ciertos instrumentos a nivel individual a todos los miembros para determinar la resiliencia familiar, lo cual no solo sería limitado, sino que no corresponde a la perspectiva sistémica. Card y Barnett (2015) proponen concebir la resiliencia familiar como un constructo que engloba cuestiones tanto individuales, familiares y contextuales, permitiendo el uso de modelos multinivel.

Por otra parte, el que la resiliencia familiar es multifactorial, hace que una escala que abarque y unifique la percepción de la familia puede ser limitada. Las mediciones transversales no ofrecen una conceptualización causal o de largo plazo. Mientras que los investigadores cuantitativos se han dedicado a aplicar escalas de medición; los investigadores cualitativos han pedido a las familias que describan su percepción sobre sus fortalezas y factores de resiliencia (Black y Lobo, 2008). "El objetivo por tanto no es desarrollar una escala de resiliencia, sino brindar una atención más longitudinal hacia nuestra consideración del estrés y fortalezas familiares con el fin de maximizar la eficacia de nuestras intervenciones" (Hawley y DeHaan, 1996, p. 66).

Como alternativa se proponen "diseños longitudinales, que capturen los procesos familiares en múltiples puntos en el tiempo, se adaptan mejor para evaluar la resiliencia que los diseños de investigación transversal, que evalúan la resiliencia familiar en un punto en el tiempo" (Hawley y DeHaan, 1996, p. 67). En la misma línea, Card y Barnett (2015) mencionan que debido a que no es práctico, ni ético, el uso de experimentos para determinar causalidad en los estudios de resiliencia se propone hacer investigaciones longitudinales, puesto que la resiliencia familiar "solo puede ser entendida como resultado de interacciones que dan forma al comportamiento a lo largo del tiempo" (p. 126). Es notable la falta de estudios longitudinales en las publicaciones en idioma español.

Así también, Card y Barnett (2015) proponen distintas alternativas a las cuestiones de confiabilidad, validez y varianza, argumentando que ya que el campo de la resiliencia familiar se ha beneficiado de distintas aproximaciones conceptuales, debe también beneficiarse de distintas aproximaciones metodológicas.

El que España sea el país con más publicaciones, pone de manifiesto la necesidad de desarrollar investigaciones sobre resiliencia familiar en los contextos latinoamericanos. Dado que se trata de un campo en crecimiento, es de suma importancia que en aras del avance científico, la elaboración de investigaciones en los contextos latinoamericanos atiendan a las recomendaciones realizadas por los expertos, desde 
las realidades de sus contextos; así como sus propias elaboraciones en este campo en desarrollo. Uno de los principales retos es incrementar programas y políticas sustentadas en las investigaciones sobre resiliencia familiar.

\section{REFERENCIAS}

Antonovsky, A. (1996). The salutogenesis model as a theory to guide health promotion. Health Promotion International, 11(1), 11-18.

Black, K. \& Lobo, M. (2008). A conceptual review of family resilience factors. Journal of Family Nursing, 14(1), 33-35.

Borges, Z., y Silva, ME. (2010). Promoción de la esperanza y resiliencia familiar. Prácticas apreciativas. Investigación y Educación en Enfermería, 28(2), 250-257.

Brendtro, L. (2006). The vision of Urie Bronfenbrenner: Adults who are crazy about kids. Reclaiming children and youth, 15(3), 162-166.

Bronfenbrenner, U. (1987). La ecología del desarrollo humano. Barcelona: Paidós.

Card, N., \& Barnett, M. (2015). Methodological considerations in studiyng individual and family resilience. Family Relations, 64, 120-133.

Cardozo, G., Dubini, P. (2005). Factores de riesgo y protección. Su incidencia en la conducta de los adolescentes. En Cardozo, G. (Coord). Adolescencia Promoción de Salud y Resiliencia (pp. 43-62). Córdoba: Brujas.

Castiblanco, J. y Lora, M. (2008). Potenciación de pautas parentales resilientes con hijos adolescentes. Revista Tendencias \& Retos, 13, (39-54).

De Andrade, M., y Da Cruz, S. (2011). Resiliencia familiar: nuevas perspectivas en la promoción y prevención en salud. Diversitas: Perspectivas en Psicología, $7(1), 43-55$.

Fernández, J., Parra, B., y Torralba, J. (2012). Trabajo social con familias y resiliencia familiar: un análisis de la relación entre ambos conceptos. Revista Atlántida, 4, 91-102.

Fitzgerald, J., Galyer, K., Whiu, G., \& Thomas, P. (2010). Understanding families and suicide risk. Auckland: Te Pou.

García-Vesga, M C. y Domínguez de la Ossa, E. (2013). Desarrollo teórico de la Resiliencia y su aplicación en situaciones adversas: Una revisión analítica. Revista Latinoamericana de Ciencias Sociales, Niñez y Juventud, 11(1), 63-77. 
Gómez, E., y Kotliarenco, MA. (2010). Resiliencia familiar: un enfoque de investigación e intervención con familias multiproblemáticas. Revista de Psicología, 19(2), 103-131.

González, C. (2004). Transformación y resiliencia en familias desplazadas por la violencia hacia Bogotá. Revista de Estudios Sociales, 18, 123-130.

Grau, C. (2013). Fomentar la resiliencia en familias con enfermedades crónicas pediátricas. Revista Española de Discapacidad, 1(1), 195-212.

Grotberg, E. (2001). Nuevas tendencias en resiliencia. En Melillo, A., Suarez, E. (comp.) Resiliencia. Descubriendo las propias fortalezas. Buenos Aires: Paidós.

Hawley, D., DeHaan, L. (1996). Toward a definition of family resilience.Integrating life-span and family perspectives. En Boss, P. Family Stress. Classic and contemporary readings (pp. 57-70). Minnesota: SAGE.

Hernán, M., y Lineros, C. (2010). Los activos para la salud. Promoción de la salud en contextos personales, familiares y sociales. Fundesfam. 2. [Citado el 20 de agosto de 2014]. Disponible en: www.fundesfam.org/REVISTA\%20 FUNDESFAM\%201/007revisiones.htm

Herscovici, C. (2011). Resiliencia familiar y el énfasis en los recursos del sistema. Cultura, Educación, Sociedad, 2(1), 105-110.

Infante, F. (2006). La resiliencia como proceso: una revisión de la literatura reciente. En Melillo, A., y Suárez, E. (comp.). Resiliencia. Descubriendo las propias fortalezas. (pp. 31-53). Buenos Aires: Paidós.

Kotliarenco, M., Cáceres, I., y Fontanilla, M. (1997). Estado de arte en resiliencia. Washington DC: Organización Panamericana de la Salud.

Kotliarenco, MA., Muñoz, MM., y Gómez, E. (2012). Procesos de resiliencia familiar ante la adversidad social: relación, organización y juego. En prensa.

Lindstrom, B., Eriksson, M. (2006). Contextualizing salutogenesis and Antonovsky in public health development. Health promotion international, 21(3), 238-244.

Mc Guckin, C \& Minton, S. (2014). From theory to practice: Two ecosystemic approaches and their applications to understanding school bullying. Australian Journal of Guidence and Councelling, 24(1), 36-48.

Minuchin, S. (1986). Familias y terapia familiar. México: Gedisa.

Nichols, W. (2013). Roads to understanding family resilience: 1920s to the twentyfirst century. EnBecvar, D. (Ed.).Handbook of family resilience (pp. 3-16). USA: Springer. 
RESILIENCIA FAMILIAR: UNA REVISIÓN SOBRE ARTICULOS PUBLICADOS EN ESPAÑOL

Patterson, J. (2002). Understanding family resilience. Journal of Clinical Psychology, $58(3), 233-246$.

Pereira, R. (2010). Trabajando con los recursos de la familia: Factores de resiliencia familiar. Sistemas familiares, 26(1), 93-115.

Ramírez-Osorio, P., y Hernández-Mendoza, E. (2012). Resiliencia familiar, depresión y ansiedad en adolescentes en situación de pobreza. Revista de Enfermería del Instituto Mexicano del Seguro Social, 20(2), 63-70.

Rutter, M. (1999). Resilience concepts and findings: implications for family therapy. Journal of Family Therapy, 21, 119-144.

Rutter, M. (2012). Resilience: Casual pathways and social ecology. En Ungar, M. (Edit.). The social ecology of resilience. A handbook of theory and practice. (pp. 33-42). New York: Springer.

Seligman, M. \& Csikszentmihalyi, M. (2000). Positive Psychology: an introduction. American Psychologist, 55(1), 5-14.

Taylor, J. (2004). Salutogenesis as a framework for child protection: literature review. Journal of advanced nursing, 45(6), 633-643.

Tomkiewicz, S. (2004). El surgimiento del concepto. En Cyrulnik, B., Tomkiewicz, S., Guérnard, T., S., Manciaux, M., y otros. El realismo de la esperanza. Testimonios de experiencias profesionales en torno a la resiliencia. (pp. 3350). Barcelona: Gedisa.

Villalba, C. (2003). El concepto de resiliencia individual y familiar. Aportaciones en la intervención social. Psychosocial Intervention, 12(3), 283-299.

Walsh, F. (2003). Family resilience: A framework for clinical practice. Family Process,42(1), 1-18. Spanish translation, Published in Journal: Sistemas Familiares, Buenos Aires, 2005.

Walsh, F. (2004). Resiliencia familiar: estrategias para su fortalecimiento. Buenos Aires: Amorrortu. 